$$
\begin{aligned}
& \text { Pontifícia Universidade Católica } \\
& \text { DO RIO DE JANEIRO }
\end{aligned}
$$

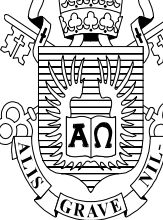

Erica dos Santos Rodrigues

\title{
Processamento da concordância de número entre sujeito e verbo na produção de sentenças
}

Tese de Doutorado

Tese apresentada ao Programa de Pós-graduação em Letras do Departamento de Letras da PUC/Rio como parte dos requisitos parciais para obtenção do título de Doutor em Letras.

Orientadora: Profa. Letícia Maria Sicuro Corrêa

Co-orientador: Prof. Jairo Morais Nunes 


$$
\text { Pontifícia Universidade } \text { Católica }_{\text {do Rio de Janeiro }}
$$

Erica dos Santos Rodrigues

\section{Processamento da concordância de número entre sujeito e verbo na produção de sentenças}

Tese apresentada como requisito parcial para obtenção do grau de Doutor pelo programa de Pós-Graduação em Letras do Departamento de Letras do Centro de Teologia e Ciências Humanas da PUC-Rio. Aprovada pela Comissão Examinadora abaixo assinada.

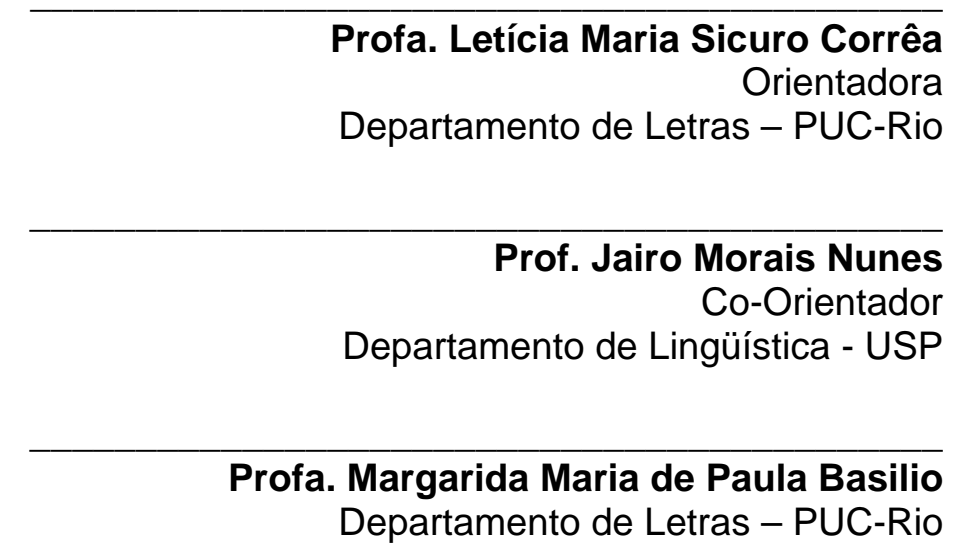

Profa. Eneida do Rêgo Monteiro Bomfim

Departamento de Letras - PUC-Rio

Prof. Marcus Antonio Rezende Maia

Departamento Antropologia - UFRJ

Profa. Mary Aizawa Kato

Departamento de Lingüística - UNICAMP

Prof. Paulo Fernando Carneiro de Andrade

Coordenador Setorial do Centro de Teologia

e Ciências Humanas - PUC-Rio

Rio de Janeiro, de de 
Todos os direitos reservados. É proibida a reprodução total ou parcial do trabalho sem autorização da universidade, da autora e da orientadora.

\section{Erica dos Santos Rodrigues}

Graduou-se em Letras (Português-Literaturas) pela UFRJ em 1991. Obteve título de Mestre em Letras pela PUC-Rio em 1996 e concluiu, em 2006, Doutorado em Letras (área de concentração: Estudos da Linguagem) na mesma instituição. Leciona na PUC-Rio desde 1992, ministrando cursos nas áreas de Comunicação e Expressão, Lingüística e Língua Portuguesa. Participa, na qualidade de pesquisadora, de projetos na área de processamento da linguagem em adultos, desenvolvidos no LAPAL - Laboratório de Psicolingüística e Aquisição da Linguagem, vinculado ao Departamento de Letras - PUC-Rio. Áreas de interesse: Psicolingüística, Ciências Cognitivas, Lingüística Teórica, Sintaxe Gerativa.

Ficha Catalográfica

Rodrigues, Erica dos Santos

Processamento da concordância de número entre sujeito e verbo na produção de sentenças / Erica dos Santos Rodrigues ; orientadora: Letícia Maria Sicuro Corrêa ; co-orientador: Jairo Morais Nunes. Rio de Janeiro : PUC, Departamento de Letras, 2006.

202 f. ; $30 \mathrm{~cm}$

Tese (Doutorado) - Pontifícia Universidade Católica do Rio de Janeiro, Departamento de Letras.

Inclui referências bibliográficas.

1. Letras - Teses. 2. Psicolingüística. 3. Processamento de sentença. 4. Produção de sentença. 5. Concordância entre sujeito e verbo. 6. Erros de atração. 7. Parser monitorador. 8. Distância linear e hierárquica. 9. Efeitos de distributividade. 10. Efeitos morfológicos. I. Corrêa, Letícia Maria Sicuro. II. Nunes, Jairo Morais. III. Pontifícia Universidade Católica do Rio de Janeiro. Departamento de Letras. III. Título. 


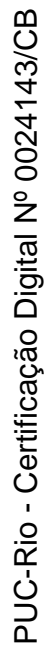

Para meu filho Henrique,

pelo olhar terno, sempre. 


\section{Agradecimentos}

A Letícia Sicuro Corrêa, minha orientadora, pelo contínuo aprendizado intelectual, pela dedicação constante, pela amizade e compreensão nos momentos críticos.

A Jairo Nunes, por ter renovado minha paixão pela sintaxe, pelo estímulo para a realização do trabalho e pelo tratamento sempre gentil e delicado.

A meu marido, pelo afeto, pela amizade, por ter acreditado mais em mim do que eu mesma.

Aos meus pais, pelo carinho e pela dedicação sem medidas.

Aos meus professores da PUC, da UFRJ e da UNICAMP, pela certeza de que estou na profissão certa.

A Cristina, Helena, Tânia, Claudinha, Vanise, Maria do Carmo, Violeta e Carmelita, por que vocês são o máximo, porque fazer tese é impossível sem amigos.

A Marina, amizade mais recente, pelas discussões de sintaxe e pela generosidade de compartilhar seu conhecimento.

A Juanito Avelar e Andrés Saab, pela troca de bibliografia e pelos comentários e sugestões.

A meu amigo-programador Claver, pela implementação dos experimentos, pelos papos, pelo exemplo de tranqüilidade.

Aos meus colegas da PUC, da UNICAMP e da UFRJ - Juanito, Ana Paula, Jéssica, Sílvia, Cínthia, Marcelo, Ricardo, Márcio, Antônio e outros, que eu possa ter esquecido de mencionar, pelo convívio e pela troca de idéias.

Aos meus colegas de trabalho, pela força na longa jornada.

Às meninas da secretaria, pelo sorriso largo e pela presteza em tudo.

A todo o pessoal do LAPAL, pela ajuda na aplicação dos experimentos e na caçada a voluntários.

A Michele, pela ajuda com os arremates da tese.

Aos participantes dos experimentos, pelos erros, meu material precioso de pesquisa. 


\section{Resumo}

Rodrigues, Erica dos Santos; Corrêa, Letícia Maria Sicuro (Orientadora); Nunes, Jairo Morais (Co-orientador). Processamento da concordância de número entre sujeito e verbo na produção de sentenças. Rio de Janeiro, 2006. 202p. Tese de Doutorado - Departamento de Letras, Pontifícia Universidade Católica do Rio de Janeiro.

O processamento da concordância de número sujeito-verbo na produção de sentenças por falantes do dialeto culto do português brasileiro é investigado. A dissertação focaliza os chamados erros de atração e seu principal objetivo é identificar os fatores que interferem no processamento da concordância e prover uma explicação psicolingüística que seja compatível com pressupostos do Programa Minimalista da Lingüística Gerativa. Mais especificamente, busca-se examinar: i) as condições que favorecem os erros e as propriedades sintáticas que levam um núcleo interveniente a ser tomado como o controlador da concordância; ii) a interferência de informação morfofonológica de número dos elementos que integram os modificadores do DP sujeito; iii) a interferência de informação semântica de número no estabelecimento da concordância. Ainda como objetivo específico busca-se distinguir em termos estruturais os DPs responsáveis pelos erros de atração daqueles que licenciam uma forma singular ou plural do verbo -- as chamadas construções partitivas. Aplica-se uma tarefa psicolingüística envolvendo julgamento de gramaticalidade a fim de investigar diferenças de processamento da concordância entre as construções partitivas e os DPs complexos. A relevância dos tópicos investigados se deve ao fato de estes permitirem uma discussão mais ampla acerca da autonomia do formulador sintático. Parte-se de vasta revisão da literatura, na qual se reportam interferências sintáticas, semânticas e morfofonológicas no processamento da concordância em diferentes línguas. Explicações apresentadas por modelos de produção interativos e não-interativos são discutidas. Inclui-se ainda uma caracterização da concepção minimalista de língua, com o tratamento da concordância como processo de valoração de traços formais, e um modelo de produção de natureza serial, não-interativo, que incorpora um parser-monitorador funcionando paralelamente à formulação dos enunciados - modelo PMP (produção monitorada por parser). Em seguida, reportam-se 5 experimentos com falantes de português. Os resultados indicaram efeito de marcação e de distância linear entre o núcleo do sujeito e o verbo, com mais erros para núcleo do sujeito não-marcado (singular) e linearmente distante do verbo, e efeito de posição estrutural do núcleo interveniente, com maior incidência de erros para os núcleos hierarquicamente próximos do nó mais alto do DP sujeito e núcleos inseridos em PPs argumentos. Um efeito semântico de distributividade associado a efeito de marcação também foi obtido. Quanto a fatores morfofonológicos, a informação de número no determinante (e não no nome) mostrou-se crucial para a identificação do número do DP sujeito. É proposta uma versão ampliada e revista do modelo PMP que unifica explicações para os erros de concordância em termos de uma escala de acessibilidade da representação do DP sujeito pela memória de trabalho e que leva em consideração as expectativas do parser como possível fator de interferência em erros de atração. Essa interferência ocorreria após o parsing do primeiro DP e afetaria a codificação morfofonológica do verbo. Em suma, a tese aqui veiculada é a de que os erros de concordância não ocorrem na computação sintática e que o formulador sintático atua de forma autônoma.

\section{Palavras-chave}

Psicolingüística; processamento de sentença; produção de sentença; concordância entre sujeito e verbo; concordância entre sujeito e verbo no português; erros de atração, parser monitorador; distância linear e hierárquica; efeitos de distributividade; efeitos morfofonológicos. 


\section{Abstract}

Rodrigues, Erica dos Santos; Corrêa, Letícia Maria Sicuro (Advisor); Nunes, Jairo Morais (Co-advisor). Processing of Subject-verb Number Agreement in Sentence Production. Rio de Janeiro, 2006. 202p. Phd Dissertation Departamento de Letras, Pontifícia Universidade Católica do Rio de Janeiro.

The processing of subject-verb number agreement in sentence production by speakers of the standard dialect of the Brazilian Portuguese is investigated. The dissertation focuses on attraction errors and its main aim is to identify the factors that interfere in agreement processing and to provide a psycholinguistic account, which is compatible with assumptions of the Minimalist Program of Generative Linguistics. This work examines, in particular: i) the conditions that favor attraction errors and the syntactic properties which make an intervenient head to be considered as the agreement controller; ii) the role of morphophonological information on number provided by the DP subject modifiers; iii) the interference of conceptual number in agreement. Additionally, the work intends to distinguish, in structural terms, the DPs responsible for attraction errors from those DPs that allow a singular and a plural form of the verb - the so-called partitive constructions. A psycholinguistic procedure of grammaticality judgment is conducted in order to verify agreement processing differences between partitive constructions and complex DPS. The relevance of these topics is due to the fact that they enable a more comprehensive discussion on the autonomy of the syntactic formulator in language production. An extensive review of the existing literature was carried out and results are reported, concerning the interference from syntactic, semantic and morphophonological factors on agreement processing in different languages. Explanations provided by interactive and non-interactive models are discussed in this work. The minimalist conception of language is presented according to which agreement is described as a feature valuation process and a serial non-interactive production model is characterized, which incorporates a monitoring-parser that works in parallel with speech formulation - PMP model (parser monitored production). A total of 5 experiments with Portuguese speakers are reported. The results show an effect of markedness and linear distance between the subject and the verb, with more errors caused by non-marked (singular) subject heads that are linearly distant from the verb, and an effect of the structural position of the intervenient head, with a large number of errors for intervenient heads that are near to the upper phrasal marker of the DP and for heads which are inserted in PP arguments. A semantic effect of distributivity associated with an effect of markedness was also obtained. As far as morphphonological factors, number information of the determiner (and not of the noun) has shown to be critical to subject number identification. A revised and improved version of the PMP model is proposed that unifies possible explanations for agreement in terms of on an accessibility scale of the DP subject representation in the working memory and that takes into account parser predictions as a possible factor of interference in attraction errors. This interference would occur after the parsing of the DP and would affect the morphophonological encoding of the verb. In sum, the main argument of the thesis is that agreement errors do not occur in the syntactic computation and that the syntactic formulator works autonomously.

\section{Keywords}

Psycholinguistics; Sentence Processing; Sentence Production; Subject-verb Agreement; Subject-verb Agreement in Portuguese; Attraction Errors; Monitoring Parser; Linear and hierarchical distance; Distributivity effects; Morphophonological Effects. 


\section{Sumário}

1. Introdução

2. Arquitetura do sistema de produção e o processamento da concordância: a autonomia do formulador sintático em modelos interativos e nãointerativos

3. Articulação entre teoria lingüística e psicolingüística no estudo do processamento da concordância

3.1. Arquitetura do sistema lingüístico e a derivação sintática da sentença no Minimalismo

3.2. Formulação sintática e derivação sintática

4. A investigação de erros de produção no estudo do processamento da concordância sujeito-verbo

4.1. Tipos de erros e fatores atuantes no processamento da concordância $\quad 37$

4.1.1. Fatores semânticos

4.1.1.1. Distributividade

4.1.1.2. Coletivos, bipartidos e pluralia tantum $\quad 42$

4.1.2. Fatores sintáticos

49

4.1.2.1. Distância linear

4.1.2.1.1. Distância linear entre o núcleo do sujeito e o verbo 49

4.1.2.1.2. Distância linear do núcleo interveniente em relação ao verbo 52

4.1.2.2. Distância hierárquica

4.1.2.3. Status argumental do sintagma modificador

4.1.2.3.1. Resolução de ambigüidades estruturais e o papel de informação relativa à estrutura argumental de nomes e verbos

4.1.2.3.2. Diferenças estruturais entre argumentos e adjuntos e questões de acessibilidade no processamento

4.1.2.3.3. Erros de atração e o papel do status argumental de PPs 79 modificadores

4.1.3. Fatores morfofonológicos

5. Distinguindo casos de concordância licenciados pela gramática da língua de falhas de processamento: uma análise da concordância "facultativa” com construções partitivas

5.1. Análise lingüística

5.1.1. Concordância “ad sensum” e leitura de grupo vs. leitura distributiva 89

5.1.2. Estrutura das partitivas e dupla possibilidade de concordância 97

$\begin{array}{lr}\text { 5.1.2.1. Análise proposta } & 106\end{array}$

5.1.2.2. A dupla possibilidade de concordância 116

5.2. Experimento psicolingüístico de julgamento de gramaticalidade construções partitivas x DPs complexos

6. Modelos interativos e não-interativos diante dos erros de atração

6.1. Modelos interativos

6.2. Modelos não-interativos 
7. Modelo PMP - modelo de produção monitorada por parser

7.1. Propriedades do modelo

7.1.1. Incrementalidade moderada

7.1.2. Computação automática da concordância como processo de valoração de traços

7.1.3. Monitoração concomitante à produção por parte de um parser

7.2. Procedimentos implementados pelo parser monitorador e explicação dos erros de atração

8. Experimentos de produção induzida de erros

8.1. Questões metodológicas no estudo da produção da linguagem e a tarefa de indução de erros

8.2. Experimentos

8.2.1. Experimento 1 - distância linear sujeito-verbo e tipo de modificador

8.2.2. Experimento 2 - posição linear vs. posição hierárquica do núcleo interveniente e marcação morfofonológica

8.2.4. Experimento 4 - distributividade e marcação morfofonológica 159

8.2.5. Experimento 5 - distributividade

9. Modelo de produção PMP revisto e ampliado

9.1. Erros de atração e escala de acessibilidade das representações geradas pelo parser

9.2. Modelo PMP e Programa Minimalista: considerações acerca de um tratamento unificado para a computação sintática da concordância

10. Síntese e considerações finais

11. Referências bibliográficas 


\section{Lista de figuras}

Figura 1: Arquitetura do sistema de produção da linguagem e monitoração da fala.

Figura 2: Concordância como operação de cópia de traços (Vigliocco, Butterworth \& Garrett, 1996).

Figura 3: Concordância como operação de unificação de traços (Vigliocco, Butterworth \& Garrett, 1996).

Figura 4: Computação da concordância através da operação Agree.

Figura 5: Esquema de percolação ascendente do traço de número do núcleo interveniente para o nó mais alto do sujeito.

Figura 6: Esquema de percolação ascendente do traço de número de núcleos intervenientes inseridos em PPs e em orações

Figura 7: Operações de Number Marking e Number Morphing no processamento da concordância sujeito-verbo (adaptado de Bock et al., 2001)

Figura 8: Modelo PMP de processamento da concordância na produção de sentenças

Figura 9: Modelo PMP revisto e ampliado (explicação 2)

Figura 10 : Modelo PMP revisto e ampliado (explicação 3) 


\section{Lista de gráficos}

Gráfico 1: Médias de respostas SIM em função do tipo de DP sujeito

Gráfico 2: Médias de respostas SIM em função do número do verbo

Gráfico 3: Médias de respostas SIM em função do tipo de DP sujeito e do número do verbo

Gráfico 4: Médias de erros de concordância em função do tipo de modificador e da distância entre o núcleo do sujeito e o verbo

Gráfico 5: Médias de erros de concordância em função do número de N1 e do número de N2

Gráfico 6: Médias de erros de concordância em função do status argumental do PP modificador

Gráfico 7: Médias de erros de concordância em função do número do DP do nome local

Gráfico 8: Médias de erros de concordância em função do tipo de expressão lingüística do DP distributivo

Gráfico 9: Médias de erros de concordância em função da distributividade do DP sujeito 


\section{Lista de tabelas}

Tabela 1:Média de erros de concordância por condição experimental

Tabela 2: Média de erros de concordância em função de Número de N1 e Número de $\mathrm{N} 2$

Tabela 3: Média de erros de concordância em função do status argumental do PP que contém o núcleo interveniente

Tabela 4: Média de erros de concordância em função do número do DP do nome local

Tabela 5: Média de erros de concordância em função do tipo de expressão lingüística de distributividade

Tabela 6: Média de erros de concordância em função da distributividade do DP sujeito

Tabela 7: Média de erros de repetição do preâmbulo em função da distributividade do DP sujeito 


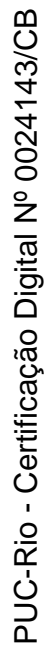

É preciso começar o desenho para saber o que se quer desenhar.

Pablo Picasso 